\title{
Thick heavily boron doped CVD diamond films homoepitaxially grown on (111)-oriented substrates
}

\author{
A. Boussadia , A. Tallairea , O. Brinza ${ }^{a}$, M.A. Pinault-Thaury ${ }^{b}, J^{\text {. Achard }}{ }^{a, *}$ \\ a LabORATOIRE des Sciences des Procédés et des MATERIAUX (LSPM), CNRS, Université PaRIS 13, \\ Sorbonne Paris Cité, 93430 Villetaneuse, France \\ b Groupe d'Etude de la Matiere Condensée (GEMAC), CNRS, Université Versailles St-Quentin-en- \\ Yvelines (UVSQ) Université Paris Saclay, 45 aVenue des Etats Unis, 78035 Versailles Cedex, France
}

\begin{abstract}
The development of diamond power electronic devices based on $p-n$ junctions strongly relies on the ability to achieve efficient $n$-type doping which has so far been the limiting step. (111)Oriented diamond films offer the advantage of a higher activity and incorporation of dopants and is considered as the most adapted orientation. Nevertheless, with a view to develop bipolar vertical components, which is the most suitable geometry for power electronic devices, the growth of thick p-type (111)-oriented substrates is essential. In this respect, growing high- quality films by Plasma Assisted Chemical Vapour Deposition (PACVD) on this orientation is critical, particularly for doping purposes since (111) CVD films are plagued by twinning and defects that are easily formed. Good quality highly boron doped (111) CVD films have been obtained but only for low thicknesses (a few $\mu \mathrm{m})$. In this paper, boron doped diamond growth was carried out on (111)-oriented high pressure high temperature sub- strates by high power PACVD with growth conditions allowing synthesis of thick and heavy boron doping. A layer with a thickness of $\sim 100 \mu \mathrm{m}$ and boron doping level of a few $10^{20}$ $\mathrm{cm}^{-3}$ has been successfully obtained opening the way to the development of (111) bipolar vertical component.
\end{abstract}

\section{Introduction}

Progress achieved in the control of microwave plasma assisted chemical vapour deposition (PACVD) diamond growth allows the quasi routine growth of millimetre-thick intrinsic diamond films $[1,2]$. Recently this process has been extended to the growth of heavily boron doped CVD films $[3,4]$ allowing highly conductive p-type (100) oriented diamond substrates that can be useful to fabricate diamond vertical power devices [5]. However, growth optimization of thick p-type and intrinsic diamond has been mainly carried out on (100) orientation even if some recent works have shown that thick highquality intrinsic diamond films can be grown on (111) as well [6]. Historically, due to the higher doping efficiency and the better donor electrical activity, (111) orientation has been mainly used for $n$-type doping with phosphorus $[7,8]$, leading to the first fabrication of bipolar components $[9,10]$. This orientation has been and is still also used for $p$-type doping with boron for achieving very high doping level (> 1× $1022 \mathrm{~cm}-3)$ particularly for superconductivity applications [11-14]. So far the related works have been limited to the growth of thin films $(<50 \mu \mathrm{m})$ which in many cases are large enough. Nonetheless, there is a strong interest in the development of a process leading to the growth of thick heavily boron doped material on (111). Indeed, it could allow the development of vertical bipolar components with better electronic properties of $n$-type layer even if it is well known that the growth of thick films on this orientation is very challenging [6]. In this paper, we will present optimization of a growth process allowing the synthesis of thick heavily boron doped films with a (111) orientation.

\section{Experimental details}

Type-lb High-Pressure-High-Temperature (HPHT) (111) oriented diamond single crystals have been used as substrates for all homoepitaxial diamond growth carried out in this study. The size of the substrates was approximately $2 \times 2 \times 0.5 \mathrm{~mm}^{3}$ and all of them have a minimum miscut angle of $2^{\circ}$ (values 
given by the supplier) since a polishing on an exact (111) orientation is particularly difficult. The crystals have been cleaned in boiling $\mathrm{HCl} / \mathrm{HNO}_{3}$ acid mixture. Starting from the growth conditions developed and optimized for (111) intrinsic material [6], a set of 5 samples has been grown using a commercial microwave CVD reactor (Plassys BJS150). Microwave power and pressure of $2.75 \mathrm{~kW} / 220 \mathrm{mbar}$ have been adjusted with a substrate temperature of $1250^{\circ} \mathrm{C}$. The gas phase typically consisted of a mixture of purified hydrogen $(9 \mathrm{~N})$, highly pure methane $(6 \mathrm{~N})$ and diborane $(6 \mathrm{~N})$. Methane concentration has been varied from 1 to $3 \%$ for all of these samples, and $\mathrm{B} / \mathrm{C}$ ratio in the gas phase has been kept constant to $1500 \mathrm{ppm}$. Diborane, which is diluted in a $\mathrm{H} 2$ cylinder at a concentration of $3000 \mathrm{ppm}$, has been adjusted accordingly in a total gas flow of $200 \mathrm{sccm}$. The growth conditions are summarized in Table 1. Grown thicknesses have been measured using a calliper. Surface morphologies have been observed using a laser microscope Keyence VK 9700 . High resolution $\left(<0.2 \mathrm{~cm}^{-1}\right)$ micro-Raman spectroscopy has been performed using a Jobin-Yvon HR800 equipment fitted with an excitation source at $632.8 \mathrm{~nm}$ having a spot diameter lower than $1 \mu \mathrm{m}$. Boron doping in diamond films has been measured by Secondary-Ion Mass Spectrometry (SIMS) with Cs+ primary ions using a Cameca IMS7f apparatus. Cathodoluminescence $(\mathrm{CL})$ spectroscopy was performed with a Jobin-Yvon HClue system attached to a Zeiss EVO-MA15 scanning electron microscope. A $10 \mathrm{kV}$ electron beam and a $20 \mathrm{nA}$ current was sent to the sample cooled down to $\sim 110 \mathrm{~K}$ thanks to a liquid nitrogen cooled stage. The light was collected by a parabolic mirror and sent to the spectrometer equipped with 600 and 1800 grooves $/ \mathrm{mm}$ diffraction gratings.

\section{Results and discussion}

The optical pictures of the films presented in the bottom row of Table 1 show that above $2 \%$ of methane in the gas phase, the surface morphology of the films begins to deteriorate with the appearance of micro-cracks (samples $\mathrm{H}$ and I). As already discussed by Tallaire et al. [6], an increase of methane concentration in the gas phase leads to an increase of the $\alpha$ parameter defined as

$$
\alpha=\sqrt{3} \frac{\mathrm{v}_{(100)}}{\mathrm{v}_{(111)}}
$$

where $v(100)$ and $v(111)$ are the growth rates of the (100) and (111) orientations. If $\alpha>1.5$, penetration twins on (111) cannot survive [15] because their growth rate towards the $\langle 111\rangle$ direction is lower than that of their parent face. For lower concentrations, all the films present a smooth appearance and are free of non-epitaxial defects and twinning. As expected, the growth rate increases with methane concentration to reach $4.3 \mu \mathrm{m} \cdot \mathrm{h}^{-1}$ at $3 \%$ against $0.95 \mu \mathrm{m} \cdot \mathrm{h}^{-1}$ at $1 \%$. It can also be noted that the increase of the growth rate seems to be quasi-linear for the 3 lowest concentrations before exponentially increasing above $2.5 \%$ as illustrated on Fig. 1 . This abrupt increase is probably related to the degradation of the surface morphology which is likely to lead to a more efficient incorporation of the carbon radicals.

Raman spectra of the films are presented in Fig. 2(a). As largely described in the literature, boron incorporation (over a few $10^{19} \mathrm{at} / \mathrm{cm}^{3}$ ) in diamond induces the appearance of a Fano effect leading to a dissymmetry of the baseline and a shift of the diamond peak towards lower frequencies [16-18]. Moreover, at high doping level (close to $10^{20} \mathrm{at} / \mathrm{cm}^{3}$ ), broad bands are generally observed in the wavelength range from 300 to $1200 \mathrm{~cm}^{-1}[17,19-21]$.

When the methane concentration increases, a reinforcement of this effect and the associated broad bands is observed which suggests an increase of the boron concentration in the films. Moreover, even with a low methane content, the Fano effect is visible, indicating that the doping level remains as high as few $10^{19} \mathrm{~cm}^{-3}[16,22]$. This trend is consistent with the results of Okushi et al. [23] and can be related to an increase of the growth rate. Indeed, it has been reported that a boron precursor adsorbed on the diamond surface has a high desorption probability [24] and consequently, an increase of the growth rate may allow the boron to be rapidly incorporated into the crystal lattice before being desorbed.

The crystalline quality of the diamond films was also evaluated from the Full Width at Half Maximum of the diamond Raman peak (FWHM). In the case of highly boron doped (100) single crystals previously synthetized, FWHM between 1.6 and $5.3 \mathrm{~cm}^{-1}$ have been reported by Issaoui et al. [22]. The FWHM on (111) oriented substrates is much higher and increases with the methane concentration from 4.9 to 20.1 $\mathrm{cm}^{-1}$. This trend may indicate a decrease in the quality of the material [25], but is more likely associated to the important Fano effect with high boron doping level [26]. In order to have a better view of these two contributions, FWHM was reported as a function of boron concentration measured by SIMS for 
samples $F$ and $G$ (Fig. 3). These results are compared with those obtained with non-intentionally doped material (NID sample in Fig. 3) grown on (111) orientation and boron doped films grown on (100) orientation [22]. We observe that the boron content is higher in (111) samples than in (100) films with doping levels ranging from 2 to $3 \times 10^{20} \mathrm{~cm}^{-3}$. Moreover, the evolution of the FWHM follows a similar trend with doping independently of the orientation. This lack of discontinuity suggests that the enlargement of the diamond Raman peak is mainly due to an increase of the doping level rather than the decrease of the crystalline quality.

The films $F$ and $G$ which present the best compromise between surface morphology and growth rate were further analysed by CL. The spectra obtained are shown in Fig. $4 \mathrm{a}$ and b. They are dominated by the recombination emission of excitons bound to boron acceptors with assistance of transverse optical phonons (BETO) which shows a characteristic doublet structure at around $250 \mathrm{~nm}(5 \mathrm{eV})[27,28]$ (Fig. 4b). Broad band emission related to the presence of defects is also visible around $500 \mathrm{~nm}$ although its intensity remains moderate in comparison to exciton emission. This confirms the good crystalline quality of the grown layers. In particular, no luminescence from NV centres expected as a sharp peak around $575 \mathrm{~nm}$ is detected but only a weak contribution from SiV (around $737 \mathrm{~nm}$ ). Silicon impurities originate from the quartz bell-jar used in the reactor chamber. At this doping level, free exciton emission is not observed and thus boron concentration cannot be estimated from the FЕто to ВЕто ratio using the empirical equation reported by Barjon et al. for lightly doped material [28].

However, the ВЕто emission peak is strongly shifted towards low energies with a large broadening which corresponds to a decrease of the bandgap energy. This is consistent with a very high boron doping level [29] which is not surprising since the incorporation efficiency of dopants is known to be much higher on (111). Taking into account the boron doping measured by SIMS, an incorporation efficiency of almost $90 \%$ can be estimated which is almost 2 orders of magnitude higher than that obtained on (100) orientation in our growth conditions [30].

This preliminary study has allowed defining a set of growth parameters making possible the combination of good crystalline quality together with high boron doping level, good surface morphology and a reasonably high growth rate $(4 \mu \mathrm{m} / \mathrm{h})$ to synthetize thick films in $<48 \mathrm{~h}$. Thus, using the growth conditions of sample $\mathrm{F}$ which is the best trade-off in terms of surface morphology and leads to a doping level of a few $10^{20} \mathrm{at} / \mathrm{cm}^{3}$, an $\sim 100 \mu \mathrm{m}$ thick sample has been grown. The morphology of this sample is presented in Fig. 5 and doesn't exhibit any non-epitaxial crystallites even if a part of the surface has fish-scale patterns while the other is smooth. This phenomenon probably results from the initial misorientation of the substrate which gradually disappears during growth.

Raman spectra have been performed in the smooth and fish-scale region. The FWHM of the diamond Raman peak is $6.3 \mathrm{~cm}^{-1}$, independently of the surface morphology which is similar to that obtained for sample F. This result shows the reproducibility of the process.

\section{Conclusion}

In this work, a set of (111) oriented boron doped diamond samples has been prepared to determine optimized growth conditions allowing a high doping level together with good surface morphology and growth rates compatible with thick films deposition. Starting from the growth condition allowing the synthesis of thick (111) oriented intrinsic diamond films, it has been shown that addition of boron in the gas phase leads to films with a smooth surface, free of non-epitaxial defects. The growth of a $100 \mu \mathrm{m}$ thick boron doped diamond film on the (111) orientation with a boron concentration of a few $10^{20} \mathrm{~cm}^{-3}$ has been carried out. This result opens the way to the development of bipolar vertical component taking advantage of the more efficient n-type doping on this specific (111) orientation. In the near future CVD freestanding boron-doped (111) substrates will be prepared and elementary $p+/ n$ and $p+/ p$ - vertical devices will be tested.

\section{Acknowledgements}

This work was financially supported by the French FUI project DIAMONIX2 (F1110024M), C'Nano lle de France (SMECAL AAP-2011) and the French Labex SEAM (Science and Engineering of Advanced Materials) (ANR 11 LABX 086, ANR 11 IDEX 05 02). 
References

[1] J. Achard, F. Silva, A. Tallaire, X. Bonnin, G. Lombardi, K. Hassouni, A. Gicquel, High quality MPACVD diamond single crystal growth: high microwave power density regime, J. Phys. D 40 (2007) 6175-6188.

[2] A. Tallaire, J. Achard, F. Silva, O. Brinza, A. Gicquel, Growth of large size diamond single crystals by plasma assisted chemical vapour deposition: recent achievements and remaining challenges, C. R. Phys. 14 (2013) 169-184.

[3] R. Issaoui, J. Achard, F. Silva, A. Tallaire, M.A. Pinault, F. Jomard, A. Tardieu, A. Gicquel, Growth of thick heavily boron-doped diamond single crystals: effect of microwave power density, Appl. Phys. Lett. 97 (2010) 182101.

[4] R. Issaoui, J. Achard, A. Tallaire, F. Silva, A. Gicquel, R. Bisaro, B. Servet, G. Garry, J. Barjon, Evaluation of freestanding boron-doped diamond grown by chemical vapour deposition as substrates for vertical power electronic devices, Appl. Phys. Lett. 100 (2012) 122109.

[5] J. Achard, R. Issaoui, A. Tallaire, F. Silva, J. Barjon, F. Jomard, A. Gicquel, Freestanding CVD boron doped diamond single crystals: a substrate for vertical power electronic devices? Phys. Status Solidi A 209 (2012) 1651-1658.

[6] A. Tallaire, J. Achard, A. Boussadi, O. Brinza, A. Gicquel, I.N. Kupriyanov, Y.N. Palyanov, G. Sakr, J. Barjon, High quality thick CVD diamond films homo- epitaxially grown on (111)oriented substrates, Diam. Relat. Mat. 41 (2014) 34-40.

[7] S. Koizumi, Growth and characterization of phosphorus doped n-type diamond thin films, Phys. Status Solidi A 172 (1999) 71-78.

[8] S. Koizumi, M. Kamo, Y. Sato, H. Ozaki, T. Inuzuka, Growth and characterization of phosphorous doped $\{111\}$ homoepitaxial diamond thin films, Appl. Phys. Lett. 71 (1997) 10651067.

[9] S. Koizumi, K. Watanabe, M. Hasegawa, H. Kanda, Ultraviolet emission from a diamond pn junction, Science 292 (2001) 1899-1901.

[10] S. Koizumi, K. Watanabe, M. Hasegawa, H. Kanda, Formation of diamond p-n junction and its optical emission characteristics, Diam. Relat. Mat. 11 (2002) 307-311.

[11] E. Bustarret, J. Kačmarčik, C. Marcenat, E. Gheeraert, C. Cytermann, J. Marcus, T. Klein, Dependence of the superconducting transition temperature on the doping level in singlecrystalline diamond films, Phys. Rev. Lett. 93 (2004) 237005.

[12] E.A. Ekimov, V.A. Sidorov, E.D. Bauer, N.N. Mel'nik, N.J. Curro, J.D. Thompson, S.M. Stishov, Superconductivity in diamond, Nature 428 (2004) 542-545.

[13] A. Kawano, H. Ishiwata, S. Iriyama, R. Okada, T. Yamaguchi, Y. Takano,

H. Kawarada, Superconductor-to-insulator transition in boron-doped diamond films grown using chemical vapor deposition, Phys. Rev. B 82 (2010) 085318.

[14] Y. Takano, M. Nagao, I. Sakaguchi, M. Tachiki, T. Hatano, K. Kobayashi, H. Umezawa, H. Kawarada, Superconductivity in diamond thin films well above liquid helium temperature, Appl. Phys. Lett. 85 (2004) 2851-2853.

[15] C. Wild, R. Kohl, N. Herres, W. Muller-Sebert, P. Koidl, Oriented CVD diamond films: twin formation, structure and morphology, Diam. Relat. Mat. 3 (1994) 373-381.

[16] F. Pruvost, A. Deneuville, Analysis of the Fano in diamond, Diam. Relat. Mat. 10 (2001) 531535.

[17] V.D. Blank, V.N. Denisov, A.N. Kirichenko, M.S. Kuznetsov, B.N. Mavrin, S.A. Nosukhin, S.A. Terentiev, Raman scattering by defect-induced excitations in boron-doped diamond single crystals, Diam. Relat. Mat. 17 (2008) 1840-1843.

[18] G. Faggio, G. Messina, S. Santangelo, G. Prestopino, I. Ciancaglioni, M. Marinelli, Raman scattering in boron-doped single-crystal diamond used to fabricate Schottky diode detectors, J. Quant. Spectrosc. Radiat. Transf. 113 (2012) 2476-2481.

[19] M. Mermoux, F. Jomard, C. Tavares, F. Omnes, E. Bustarret, Raman characteriza- tion of boron-doped $\{111\}$ homoepitaxial diamond layers, Diam. Relat. Mat. 15 (2006) 572-576.

[20] M. Bernard, A. Deneuville, P. Muret, Non-destructive determination of the boron concentration of heavily doped metallic diamond thin films from Raman spectro- scopy, Diam. Relat. Mat. 13 (2004) 282-286.

[21] M. Bernard, C. Baron, A. Deneuville, About the origin of the low wave number structures of the Raman spectra of heavily boron doped diamond films, Diam. Relat. Mat. 13 (2004) 896-899.

[22] R. Issaoui, PhD Thesis, 13 University Paris, 2011.

[23] H. Okushi, High quality homoepitaxial CVD diamond for electronic devices, Diam. Relat. Mat. 10 (2001) 281-288.

[24] M. Ogura, H. Kato, T. Makino, H. Okushi, S. Yamasaki, Misorientation-angle dependence of boron incorporation into (001)-oriented chemical-vapor-deposited (CVD) diamond, J. Cryst. 
Growth 317 (2011) 60-63.

[25] S.J. Harris, A.M. Weiner, S. Prawer, K. Nugent, Diamond film quality: effects of gas phase concentrations on the Raman spectra, J. Appl. Phys. 80 (1996) 2187-2194.

[26] U. Fano, Effects of configuration interaction on intensities and phase shifts, Phys. Rev. 124 (1961) 1866-1878.

[27] C. Tavares, F. Omnes, J. Pernot, E. Bustarret, Electronic properties of boron-doped \{111\}oriented homoepitaxial diamond layers, Diam. Relat. Mat. 15 (2006) 582-585.

[28] P. Dean, Bound excitons and donor-acceptor pairs in natural and synthetic diamond, Phys. Rev. 139 (1965) A588.

[29] C. Baron, M. Wade, A. Deneuville, F. Jomard, J. Chevallier, Cathodoluminescence of highly and heavily boron doped (100) homoepitaxial diamond films, Diam. Relat. Mat. 15 (2006) 597601.

[зо] J. Achard, F. Silva, R. Issaoui, O. Brinza, A. Tallaire, H. Schneider, K. Isoird, H. Ding, S. Kone, M.A. Pinault, F. Jomard, A. Gicquel, Thick boron doped diamond single crystals for high power electronics, Diam. Relat. Mater. 20 (2011) 145-152.

Table 1: Growth conditions and main characteristics of the boron doped diamond films grown on (111) orientation with the same $B / C$ ratio of $1500 \mathrm{ppm}$.
Samples
E $F$
G $\quad H$

1

$\begin{array}{lllllr}\text { DсH4 }(\mathrm{sccm}) & 2 & 3 & 4 & 5 & 6 \\ \mathrm{D}_{\text {B2H6 }}(\mathrm{sccm}) & 0.5 & 0.75 & 1 & 1.25 & 1.5 \\ \% \mathrm{CH}_{4} & 1 & 1.5 & 2 & 2.5 & 3 \\ \text { Thickness }(\mu \mathrm{m}) & 22 & 29 & 33 & 53 & 90 \\ \text { Growth rate }\left(\mu \mathrm{m} \cdot \mathrm{h}^{-1}\right) & 0.95 & 1.25 & 1.45 & 2.3 & 4.3 \\ \text { Raman FWHM }\left(\mathrm{cm}^{-1}\right) & 4.9 & 6.8 & 9.0 & 15.3 & 20.1\end{array}$

Optical pictures of the gies

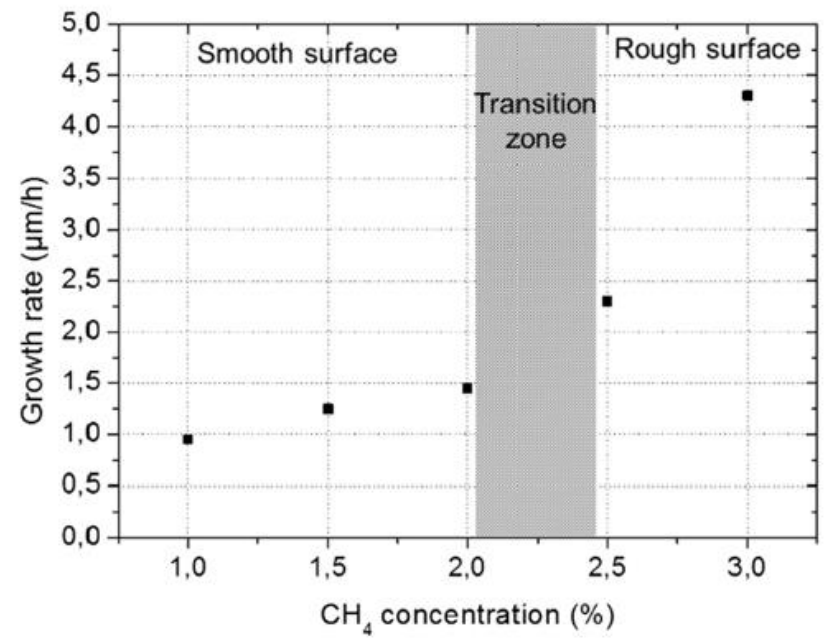

Fig. 1. Evolution of the growth rate as a function of the $\mathrm{CH}_{4}$ concentration introduced in the gas phase. 


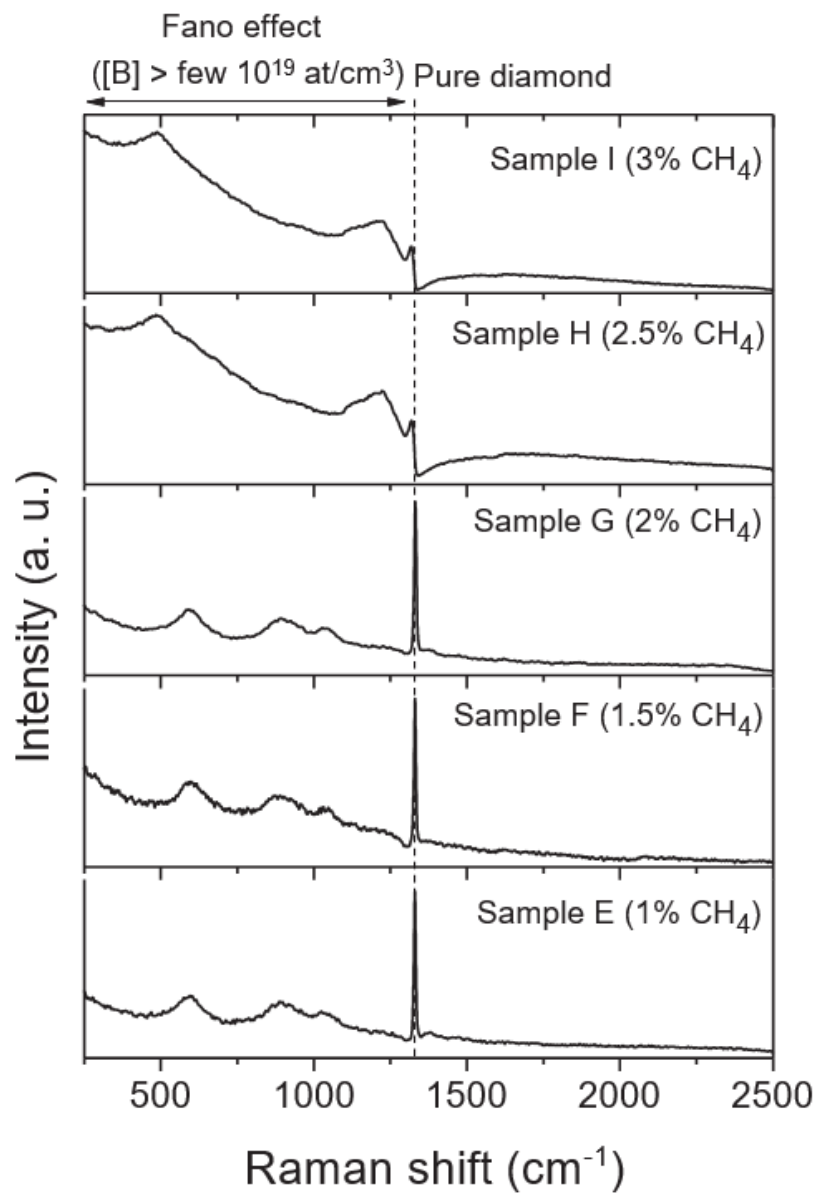

Fig. 2. Raman spectra of the boron doped layers grown on (111) orientation.

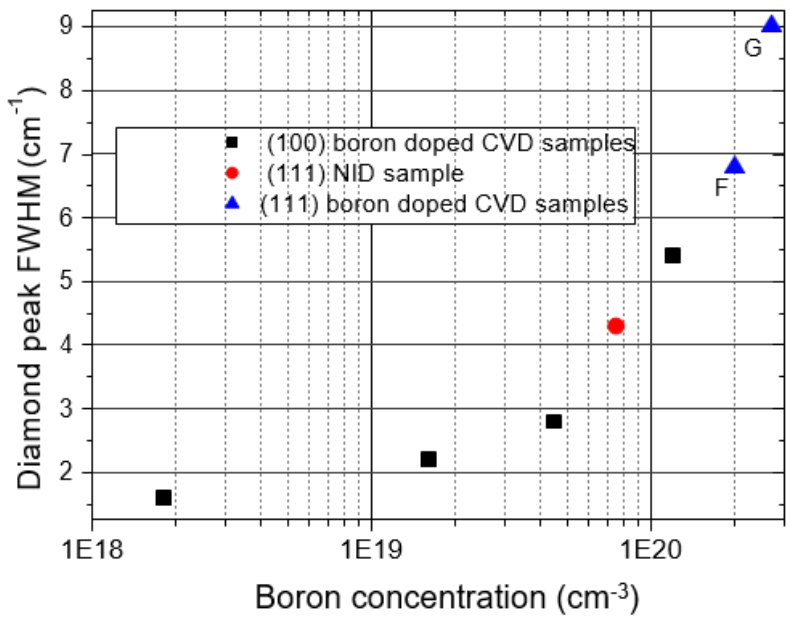

Fig. 3. Evolution of the Raman diamond peak FWHM as a function of boron doping level for samples grown on (111) and (100) orientations. 


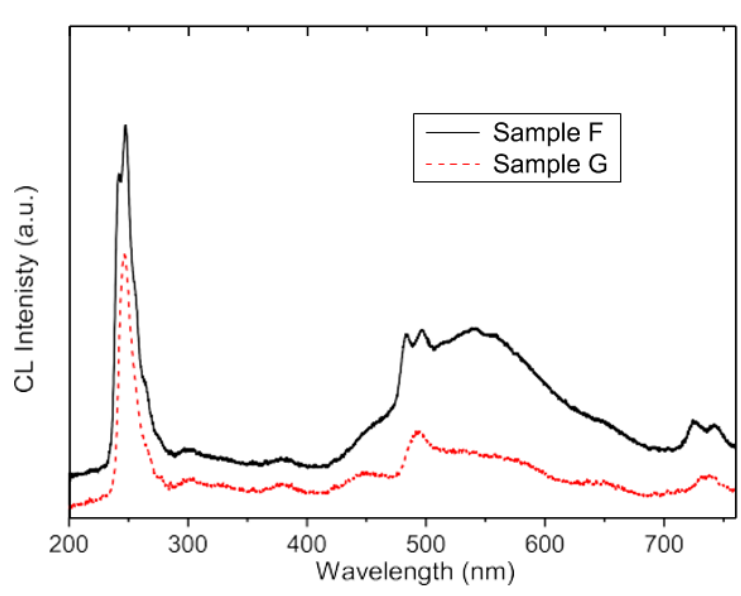

(a)

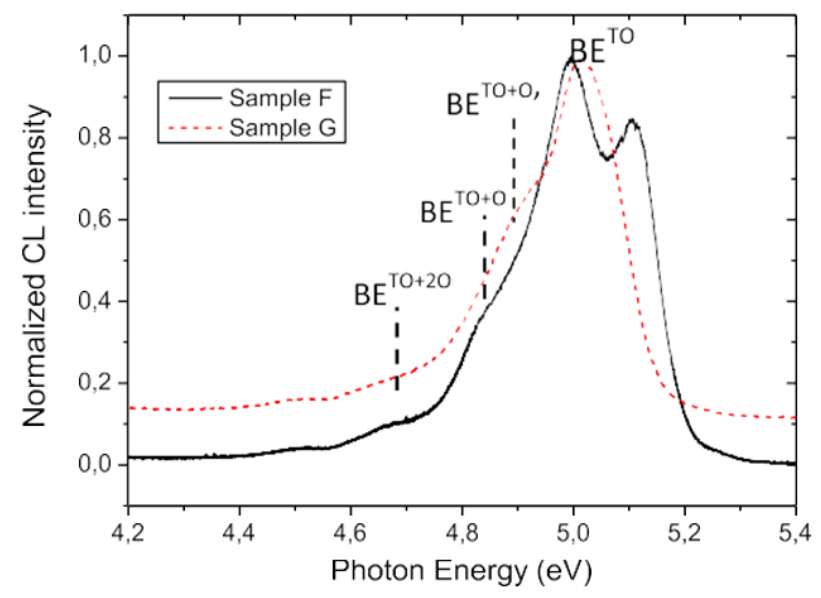

(b)

Fig. 4. Cathodoluminescence spectra of $F$ and $G$ samples that have a boron content of $2 \times 10^{20}$ $\mathrm{at} / \mathrm{cm}^{3}$ and $2.5 \times 10^{20} \mathrm{at} / \mathrm{cm}^{3}$, respectively: (a) full range and (b) zoom in the excitonic region.

BE $^{\mathrm{TO}}$ refers to neutral-boron bound exciton recombination assisted by transverse optic phonons. The exciton recombinations are assisted by phonons referred as $\mathrm{TO}, \mathrm{TO}+\mathrm{O}$ and $\mathrm{TO}+2 \mathrm{O}$.

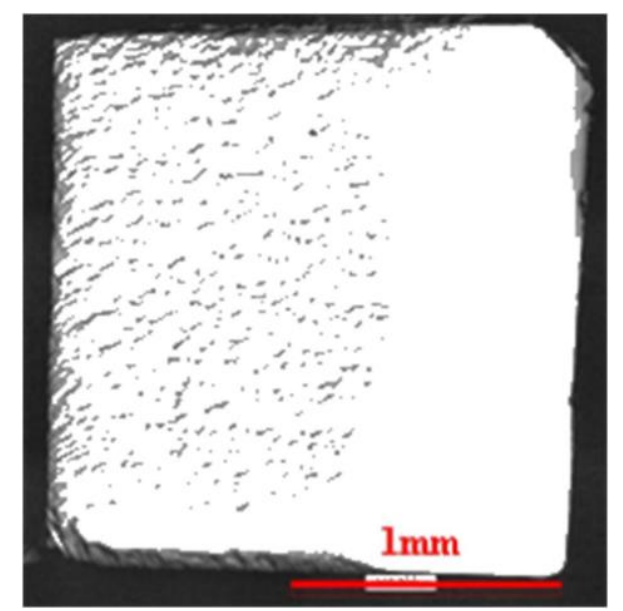

Fig. 5. Surface morphology by optical microscopy of the thick heavily boron doped sample grown on (111) oriented substrate $\left(\sim 100 \mu \mathrm{m}\right.$ with $[B] \sim$ few $\left.10^{20} \mathrm{at} / \mathrm{cm}^{3}\right)$. 